\title{
CT Texture Analysis of Ex Vivo Renal Stones Predicts Ease of Fragmentation
}

\section{with Shockwave Lithotripsy}

\section{Authors and affiliations}

HW Cui ${ }^{1 * \ddagger}$, W Devlies $^{2^{*}}$, S Ravenscroft ${ }^{3}, \mathrm{H} \mathrm{Heers}^{1,4}$, A. Freidin ${ }^{5}$, RO Cleveland ${ }^{6}$, B Ganeshan ${ }^{7}, \mathrm{BW}$

Turney ${ }^{1}$

${ }^{1}$ Oxford Stone Group, University of Oxford, Oxford, United Kingdom

${ }^{2}$ Faculty of Medicine, KU Leuven, Leuven, Belgium.

${ }^{3}$ Medical Sciences Division, University of Oxford, Oxford, United Kingdom

${ }^{4}$ Department of Urology and Paediatric Urology, Philipps-Universität Marburg, Marburg, Germany

${ }^{5}$ Kennedy Institute of Rheumatology, University of Oxford, Oxford, United Kingdom

${ }^{6}$ Institute of Biomedical Engineering, Department of Engineering Science, University of Oxford, Oxford, United Kingdom

${ }^{7}$ Institute of Nuclear Medicine, Division of Medicine, University College London, London, United Kingdom

*These authors contributed equally to this work

$¥$ Corresponding author

\section{Contact details}

\section{Helen W Cui (corresponding author)}

Address: Urology Department, Churchill Hospital, Old Road, Oxford, United Kingdom

Telephone number: 07901827404 Fax number: n/a E-mail: helen.cui@nds.ox.ac.uk

\section{Wout Devlies}

Address: Urology Department, Churchill Hospital, Old Road, Oxford, United Kingdom 
Telephone number: +32472435275 Fax number: n/a E-mail: wout.devlies@student.kuleuven.be

\section{Samuel Ravenscroft}

Address: Osler House, John Radcliffe Hospital, Osler Road, OX3 9BL

Telephone number: 07784563881 Fax number: n/a E-mail: samuel.ravenscroft@gtc.ox.ac.uk

\section{Hendrik Heers}

Address: Universitätsklinikum Marburg, Klinik für Urologie und Kinderurologie, Baldingerstraße, 35033 Marburg, Germany

Telephone number: +4964215869405 Fax:+4964215865590 E-mail: heers@med.uni-marburg.de

\section{Andrew J Freidin}

Address: Kennedy Institute of Rheumatology, University of Oxford, Roosevelt Drive, Headington, Oxford, OX3 7FY, UK

Telephone number: 07881995546 Fax number: n/a E-mail: andrew.freidin@kennedy.ox.ac.uk

\section{Robin O Cleveland}

Address: Institute of Biomedical Engineering, University of Oxford, Old Road Campus Research Building, Oxford OX3 7DQ, UK

Telephone number: 01865617737 Fax number: n/a E-mail: robin.cleveland@eng.ox.ac.uk

\section{Balaji Ganeshan}

Address: Institute of Nuclear Medicine, 5th Floor Tower, University College Hospital, 235 Euston Road

Telephone number 02034470535 Fax number: 02034470598 E-mail: b.ganeshan@ucl.ac.uk 


\section{Benjamin W Turney}

Address: Urology Department, Churchill Hospital, Old Road, Oxford, United Kingdom

Telephone number: 0186534444 Fax number: 01865572271. E-mail: ben.turney@nds.ox.ac.uk 


\section{Abstract}

\section{Introduction}

Understanding the factors affecting success of shock wave lithotripsy (SWL) would improve informed decision-making on the most appropriate treatment modality for an individual patient. Although stone size and skin-to-stone distance do correlate with fragmentation efficacy, it has been shown that stone composition and architecture, as reflected by structural heterogeneity on $\mathrm{CT}$, are also important factors. This study aims to determine if CT Texture Analysis (CTTA) - a novel, nondestructive, and objective tool that generates statistical metrics reflecting stone heterogeneitycould have utility in predicting likelihood of SWL success.

\section{Method}

7 spontaneously passed, intact, renal tract stones, were scanned ex vivo using standard CT KUB and micro computed tomography. The stones were then fragmented in vitro using a clinical lithotripter, after which, chemical composition analysis was performed. CTTA was used to generate a number of metrics which were correlated to the number of shocks needed to fragment the stone.

\section{Results}

CTTA metrics reflected stone characteristics and composition, and predicted ease of SWL fragmentation. The strongest correlation with number of shocks required to fragment the stone was mean HU density ( $r=0.806, p=0.028)$ and a CTTA metric measuring the entropy of the pixel distribution of the stone image $(r=0.804, p=0.039)$. Using multiple linear regression analysis, the best model showed that CTTA metrics of entropy and kurtosis could predict $92 \%$ of the outcome of number of shocks needed to fragment the stone. This was superior to using stone volume or density.

\section{Conclusions}


CTTA metrics entropy and kurtosis have been shown in this experimental ex vivo setting to strongly predict fragmentation by SWL. This warrants further investigation in a larger clinical study for the contribution of $\mathrm{CT}$ textural metrics as a measure of stone heterogeneity, along with other known clinical factors, to predict likelihood of SWL success. 


\section{Introduction}

Extracorporeal shock wave lithotripsy (SWL) has a reported success rate of $35-89 \%{ }^{1-5}$ and is the firstline treatment in patients with urinary calculi less than $2 \mathrm{~cm}$ within the renal pelvis and upper or middle calices. ${ }^{6,7}$ The success rate has been shown to be affected by a number of factors including stone Computed Tomography (CT) Hounsfield unit (HU) density, stone volume, stone location and

skin-to-stone distance. ${ }^{8,910,11}$ In addition to these factors, the stone's internal structure and degree of heterogeneity has been shown to affect stone fragility during SWL..$^{8,12-14}$

CT texture analysis (CTTA) allows processing of existing CT images for assessing internal structural heterogeneity. In the field of oncology, CTTA has been validated as a risk stratification tool and prognostic indicator in the evaluation of a number of tumor applications e.g. solid tumors of the lung and colon. ${ }^{15,16}$ The main advantage of CTTA is the generation of quantitative measures of heterogeneity within a region of interest (e.g. a suspicious lesion) through detailed statistical analysis of voxel distribution, as well as the use of different sized filters to enhance structures of interest (e.g. increased vascularization).

The aim of this study is to translate CTTA methods that have been successfully applied in tumor analysis to a novel use in renal tract stone analysis. CTTA metrics, determined from conventional CT images, quantify the degree of heterogeneity within a stone and these metrics will be correlated with stone fragmentation during SWL. Furthermore, this study utilizes micro computed tomography (micro-CT) of the stone to aid visualisation of the variability in stone architecture. Our hypothesis is that CTTA metrics will improve the prediction of SWL outcomes over traditional clinical factors. This could be used to help decide if a stone is a good candidate for SWL or whether alternative treatments should be considered. 


\section{Materials and Methods}

\section{Imaging of stones}

7 spontaneously passed kidney stones varying in size from $3 \mathrm{~mm}$ to $6 \mathrm{~mm}$ were provided voluntarily by 5 different patients. These 7 stones were placed into a piece of polystyrene, which held them in a fixed orientation and has minimal X-ray contrast, and scanned using a conventional CT scanner (Lightspeed VCT, GE medical systems) on standard non-contrast CT KUB settings $(0.625 \mathrm{~mm}$ slice thickness and pixel size of $0.5 \mathrm{~mm}$ by $0.5 \mathrm{~mm}$ ) and the data was exported as HU into DICOM (Digital Imaging and Communications in Medicine) files. Imaging was done in three orthogonal orientations by rotating the stone holders. This was to check assumptions regarding use of the largest slice in one plane to represent the heterogeneity characteristics of the stone as a whole.

The seven stones were also scanned in a micro-CT system (Skyscan 1174, Bruker MicroCT) in one orientation to generate an isostropic volumetric data set at $6.95 \mu \mathrm{m}$ resolution. The volumetric data was then exported as a sequence of bit-map image files which were then imported into MATLAB (The MathWorks, Inc., Natick, Massachusetts, US) using the "imread" command and analyzed using the image processing tools. Selected slices were then exported from MATLAB as a DICOM file so that it could be imported into the CTTA package.

\section{CT Textural Analysis - CTTA}

Macro-CT data was analyzed using a proprietary, commercially available CTTA software package TexRad (TexRAD research software - TexRAD Ltd www.texrad.com, part of Feedback Plc, Cambridge, UK). The software imported data from DICOM files and calculated a series of statistics based on individual pixel data points within a given region of interest (ROI) i.e. the area delineated by the stone, to generate a set of textural metrics. ${ }^{17} \mathrm{~A}$ conservative ROI was drawn manually just inside the border of each stone on high magnification of the CT image. Therefore no areas outside the stone 
were included in the ROI to minimize the partial volume effects at the border of the stone. The software then calculated the following metrics: entropy (a measure of texture irregularity), skewness (higher order statistic quantifying asymmetry of the histogram) and kurtosis (higher order statistic quantifying peakedness/sharpness/pointedness of the histogram). ${ }^{17}$ In addition, the mean (average HU intensity), standard deviation (SD - width of the histogram or dispersion from the mean) and mean of the positive pixels (average HU intensity of the positive pixels only, as some stones had areas of negative HU values) were also reported. Micro-CT data did not undergo CTTA for these metrics as the software is designed for use with macro-CT DICOM data analysis, and this would be the expected imaging modality used in clinical practice.

\section{Estimation of stone volume and density}

The volume of each stone was determined from the micro-CT data set. The total voxel count of each stone was multiplied by the voxel size of $6.5 \mu \mathrm{m}$. The mean HU density of the stone was calculated by summing the $\mathrm{HU}$ value of all voxels in the stone and then dividing by the total number of voxels.

\section{Shockwave lithotripsy}

After CT imaging, the stones underwent in vitro SWL using a clinical electromagnetic lithotripter (SLXF2, Storz Medical). Each stone was placed in a plastic tube with a conical bottom onto which was bonded a mesh with grid size of $2 \mathrm{~mm}$. The conical tube was placed in a Perspex tank containing degassed water that was mounted on the lithotripter. The tank was fitted with a thin LDPE plastic film on the bottom to allow SW transmission from the therapy head into the tank. ${ }^{18}$ The lithotripter was operated at energy level 6, with a precise focus and fired at $1.5 \mathrm{~Hz}$ which is the standard clinical treatment protocol used at our center. As the stones were ex vivo, relatively few shocks were needed to fragment each stone at the energy level usually used for patient treatment. The stone was

observed manually during SWL treatment. Fragmented stone pieces less than $2 \mathrm{~mm}$ would fall 
through the mesh into a finger cot that was placed around the tube to collect the fragments. The treatment was stopped when all fragments had passed through the mesh, at which point, the number of shocks was recorded. After SWL was performed, all stone fragments were collected and sent for stone composition analysis using Fourier transform infrared spectroscopy (FTIR).

\section{Statistical Analysis}

Statistical analysis was performed using SPSS version 22.0.0. The relationship between CTTA metrics and the number of shock waves required for fragmentation was analyzed by computing Pearson's correlation coefficient. The predictive ability of stone volume, mean HU density and CTTA metrics was analyzed using multiple linear regression modelling (using the 'Enter' method) with the outcome variable being the number of shocks required to fragment the stone. Regression diagnostics were used to check that assumptions of linearity, multicollinearity, independence of errors, homoscedasticity and normally distributed errors were not violated.

\section{Results}

\section{Stone composition and CT appearance}

Figure 1 shows the stone characteristics including stone composition, CTTA metrics and representative 2D slices from both macro-CT and micro-CT. Although the ex vivo stones look very comparable on macro-CT with each other, the micro-CT data revealed the detailed internal structure and individual differences across the seven stones.

\section{Relationship between stone architecture and CTTA metrics}

The CTTA metrics represented in Figure 1 were obtained from the ROI drawn on the largest image slice available for the stone in one orientation. Validity of use of the largest slice in one plane for CTTA was checked by comparing the largest slice in each plane to the other planes, as well as 
comparison of each slice above and below the large slice to the largest slice, to check that CTTA metrics did not differ significantly between these images. Of note, stone 5 , although a uric acid stone is markedly different in appearance compared to the other two uric acid stones ( 2 and 3 ) on both macro- and micro-CT. It appears more porous with pockets of air, which has a HU value of -1000 . As all pixels within a ROI are included in the CTTA analysis, this could result in stone 5 having a negative mean HU value. The more porous architecture may explain why stone 5 required disproportionately fewer shocks to fragment than expected for its size. Stone 5 also had a positive kurtosis, in contrast to the negative kurtosis values of all of the other stones, which is likely due to the effect of the negative HU pixels.

\section{Correlation of stone characteristics with ease of fragmentation by SWL}

Correlations were considered among all the CTTA metrics (Table 1). There was a strong positive correlation between the number of shocks required to fragment the stone and stone volume $(r=0.768, n=7, p=0.044)$ (Figure 2), as well as mean $\mathrm{HU}$ (as measured by CTTA) $(r=0.775, p=0.041)$ and mean of the positive pixels $(r=0.775, p=0.05)$. The strongest correlation with the number of shocks required to fragment the stone was CTTA measured entropy ( $r=0.804, p=0.039)$ (Figure 2). Mean HU density was also significantly positively correlated to number of shocks required to fragment the stone $(r=0.806, p=0.028)$.

\section{Predicting ease of SWL fragmentation using linear regression analysis}

Stone volume, mean $\mathrm{HU}, \mathrm{HU}$ density and CTTA measured metrics were used as predictors in multiple linear regression analysis for the outcome of number of shocks needed to fragment the stone. A multiple linear regression model using the "enter" method found that the CTTA metrics entropy and kurtosis explained a significant amount of variance (92\%) in the number of shocks needed to fragment the stone $(F(2,4)=23.3, p=0.006$, $R$ Square $=0.921$, adjusted $R$ Square $=0.881)$ (Figure 3). In 
this model, both entropy $(\beta=0.887, t(6)=6.234, p=0.003)$ and kurtosis $(\beta=-0.531, t(6)=-3.732, p=0.020)$ significantly predicted the number of shocks required. These two predictors can be summarized using the equation: 'Number of shocks needed to fragment the stone $=141^{*}$ entropy $-86.5 *$ kurtosis $-568.6^{\prime}$.

This model explained a greater degree of variance in the number of shocks needed to fragment the stone than a linear regression model using entropy $(F(1,5)=9.113, p=0.029, R$ Square $=0.646)$, stone volume $(F(1,5)=7.194, p=0.044$, $R$ Square $=0.590)$, mean $H U(F(1,5)=7.498, p=0.041, R$ Square $=0.600)$ of the stone, or mean $\mathrm{HU}$ density alone $(F(1,5)=9.298, p=0.028, R$ Square $=0.650)$.

\section{Discussion}

In this experimental model of SWL using spontaneously passed, intact, ex vivo renal tract stones, we have identified CTTA parameters reflecting stone structure heterogeneity through differences in pixel distribution. These parameters show greater predictive ability for stone fragmentation by SWL than using standard measures of stone structure alone (stone volume, mean HU and HU density).

Stone mean $\mathrm{HU}$ reflects stone composition in this study, as calcium oxalate and calcium phosphate stones were the most dense (mean $\mathrm{HU}>500$ ), followed by cystine (mean $\mathrm{HU}>300$ ) and then uric acid stones (mean HU <105). However, stones of similar composition can have visually different architecture as can be seen for stones 6 and 7. Although these are both cystine stones, stone 6 is more heterogeneous in structure, showing an irregular contour on micro-CT (Figure 1). This is reflected in the lower mean $\mathrm{HU}$ and $\mathrm{HU}$ density for stone 6 than stone 7. One would expect stone 7 to require more shocks to fragment than stone 6 based on its higher HU density, but stone 7 actually required a lower number of shocks. This ease of fragmentation is reflected in the higher entropy 
despite the lower HU density, suggesting it has additional predictive value. It is well known that different stone compositions have different susceptibilities to SWL fragmentation, and that stone composition can sometimes be inferred from the mean $\mathrm{HU}$ range that can be expected of a certain stone type. However, for patients with a new presentation of symptomatic renal tract stones, being able to predict the susceptibility of a particular stone to SWL fragmentation without prior knowledge of expected stone composition or analysis of stones already passed would be clinically useful. Stones from a range of compositions were chosen in this small study to demonstrate the proof of principle that CT texture analysis can provide additional information on stone architecture. This will form the basis for in vivo studies comprising of large cohorts of patients undergoing SWL, to investigate whether textural analysis can help determine SWL success in the clinical setting when the stone composition is yet unknown and micro-CT imaging is not possible.

A multiple linear regression model with CTTA metrics of entropy and kurtosis can account for $92 \%$ of the outcome of how many shocks are needed to fragment the stones in this study. These metrics, derived from well-known statistical concepts to objectively measure stone heterogeneity, offer a rational predictor of successful fragmentation with SWL. Entropy is a measure of randomness of the pixel distribution within the stone, ${ }^{19}$ and a large dataset of in vivo renal tract stones in a previous study from our group has shown it to be positively and nonlinearly correlated with stone volume. ${ }^{20}$ From the regression analysis in this study, entropy shows greater predictive ability for the number of shock waves needed to fragment a stone than stone volume or HU density alone, suggesting entropy is contributing additional information regarding stone heterogeneity.

Kurtosis is a measure of the combined weight of the tails of the distribution relative to the rest of the distribution. In this study, kurtosis is negatively correlated with number of shocks required to fragment the stone. This relationship supports our previous work on a clinical dataset of 126 CT KUB 
images of in vivo renal tract stones which showed a more negative kurtosis value was associated with greater likelihood of stone clearance success by SWL. ${ }^{20}$ The stones in this study that required a greater number of shocks had a more negative kurtosis (Table 2). This indicates a distribution of pixels with relatively lighter tails, which can be inferred as stone pixel distribution with fewer extreme deviations in $\mathrm{HU}$ value. This may suggest that these stones have more overall heterogeneity and are therefore more susceptible to SWL fragmentation.

Although the fragmentation tests were performed in vitro in this study, and therefore did not reflect all the factors affecting clinical SWL, it has the advantage of isolating the effects of stone characteristics alone on ease of fragmentation. This would explain the high predictive value of stone texture features for the number of shocks needed to fragment the stone. The advantage of CTTA over previous studies investigating stone structure is the use of both objective and automated measures of stone structure that can be performed on standard CT KUB, on all of the available pixel data within a stone image to reflect overall heterogeneity, and that do not rely on subjective visual inspection or chemical analysis of stone composition. ${ }^{9,12,21,22}$ In comparison to another study that has examined the relationship of stone texture to ease of fragmentation with SWL ex vivo, our study shows a stronger relationship and better predictive ability (adjusted R Squared of 0.881 compared to 0.58) using our CTTA metrics. ${ }^{14}$ In combination with this study, the validity of using CTTA deserves further investigation using in vivo stone images and with consideration of other clinical factors affecting SWL success.

With regard to the reliability of CTTA across different CT scanners with different acquisition parameters, previous studies have demonstrated that the texture analysis technique used in this study has been technically validated in a number of previous oncological and phantom studies. 
Specifically these texture parameters have shown to be less impacted by variation in image acquisitions ${ }^{23}$ and shown to be reproducible from test-retest studies. ${ }^{24,25}$

This study used ex vivo stones and therefore differences in image acquisition and resolution are to be expected between ex vivo and in vivo scanning of renal tract stones. However, in a retrospective clinical study, we have shown that the likelihood of achieving stone clearance after SWL of stone with mean size of $11.5 \mathrm{~mm}$ can be predicted using CTTA metrics with $72 \%$ overall accuracy. ${ }^{20}$ Therefore, the relationship between CTTA metrics and ease of SWL fragmentation still holds when using in vivo stone images and this study adds evidence to the proof of concept of stone textural analysis. Further work should focus on in vivo stone images and SWL fragmentation in clinical practice to best inform future pragmatic use of CTTA. This study provides evidence for proof of concept, but clearly the scanning and SWL conditions for these ex vivo stones cannot be representative of in vivo stone imaging and SWL treatment.

\section{Conclusions}

This study has identified new metrics of stone structure, as measured by CTTA generated metrics of entropy and kurtosis, which, in this experimental SWL setting, strongly predict the number of shocks required for stone fragmentation. It is not possible draw firm conclusions on the utility of CTTA on the basis of this small ex vivo study, but the results support a proof of principle that CTTA metrics reflect stone architecture when analysed alongside micro-CT images and stone composition analysis. A larger clinical study is currently underway at our center, for the contribution of CT textural metrics, along with other known clinical factors, to predict likelihood of SWL success. We hope to develop a composite scoring system, using statistical modelling of these variables in a large dataset, that clinicians can then use as part of a clinical decision making tool when counselling patients. The aim of this would be to increase the success rate of SWL by reducing the number of SWL sessions needed if 
the stone is identified to be more susceptible to SWL, as well as to counsel patients to surgery if SWL is unlikely to be successful.

\section{Author Disclosure Statement}

One author (B. Ganeshan) is a director, part-time employee and shareholder of Feedback Plc (Cambridge, England, UK), company that develops and markets the TexRAD texture analysis algorithm described in this manuscript.

All other authors declare no competing financial interests exist. 


\section{References}

1. Coz F, Orvieto M, Bustos M, et al. Extracorporeal shockwave lithotripsy of 2000 urinary calculi with the modulith SL-20: success and failure according to size and location of stones. J Endourol 2000;14(3):239-46.

2. Al-Ansari A, As-Sadiq K, Al-Said S, Younis N, Jaleel OA, Shokeir AA. Prognostic factors of success of extracorporeal shock wave lithotripsy (ESWL) in the treatment of renal stones. Int Urol Nephrol 2006;38(1):63-7.

3. Abdel-Khalek M, Sheir KZ, Mokhtar AA, Eraky I, Kenawy M, Bazeed M. Prediction of success rate after extracorporeal shock-wave lithotripsy of renal stones A multivariate analysis model. J Urol 2004;38(2):161-7.

4. Pearle MS, Lingeman JE, Leveillee R, et al. Prospective, randomized trial comparing shock wave lithotripsy and ureteroscopy for lower pole caliceal calculi 1 cm or less. J Urol 2005;173(6):20059.

5. Sumino $\mathrm{Y}$, Mimata $\mathrm{H}$, Tasaki $\mathrm{Y}$, et al. Predictors of lower pole renal stone clearance after extracorporeal shock wave lithotripsy. J Urol 2002;168(4 Pt 1):1344-7.

6. Türk C., Knoll T., Petrik A., Sarica K., Skolarikos A., Straub M., Seitz C. EAU Guidelines on Urolithiasis [Internet]. European Association of Urology; 2016. Available from: http://dx.doi.org/10.1159/000449194

7. Assimos D, Krambeck A, Miller NL, et al. Surgical management of stones: American Urological Association / Endourological Society Guideline [Internet]. American Urological Association (AUA); 2016. Available from: http://dx.doi.org/10.4135/9781412963855.n93

8. El-Nahas AR, El-Assmy AM, Mansour O, Sheir KZ. A prospective multivariate analysis of factors 
predicting stone disintegration by extracorporeal shock wave lithotripsy: the value of highresolution noncontrast computed tomography. Eur Urol 2007;51(6):1688-93.

9. Massoud AM, Abdelbary AM, Al-Dessoukey AA, Moussa AS, Zayed AS, Mahmmoud O. The success of extracorporeal shock-wave lithotripsy based on the stone-attenuation value from non-contrast computed tomography. Arab J Urol 2014;12(2):155-61.

10. Nakasato T, Morita J, Ogawa Y. Evaluation of Hounsfield Units as a predictive factor for the outcome of extracorporeal shock wave lithotripsy and stone composition. Urolithiasis 2015;43(1):69-75.

11. Ruggera L, Beltrami P, Ballario R, Cavalleri S, Cazzoletti L, Artibani W. Impact of anatomical pielocaliceal topography in the treatment of renal lower calyces stones with extracorporeal shock wave lithotripsy. Int J Urol 2005;12(6):525-32.

12. Zarse CA, Hameed TA, Jackson ME, et al. CT visible internal stone structure, but not Hounsfield unit value, of calcium oxalate monohydrate (COM) calculi predicts lithotripsy fragility in vitro. Urol Res 2007;35(4):201-6.

13. Williams JC Jr, Paterson RF, Kopecky KK, Lingeman JE, McAteer JA. High resolution detection of internal structure of renal calculi by helical computerized tomography. J Urol 2002;167(1):3226.

14. Ferrero A, Montoya JC, Vaughan LE, et al. Quantitative Prediction of Stone Fragility From Routine Dual Energy CT: Ex vivo proof of Feasibility. Acad Radiol 2016;23(12):1545-52.

15. Ganeshan B, Panayiotou E, Burnand K, Dizdarevic S, Miles K. Tumour heterogeneity in non-small cell lung carcinoma assessed by CT texture analysis: a potential marker of survival. Eur Radiol 2012;22(4):796-802. 
16. Ng F, Ganeshan B, Kozarski R, Miles KA, Goh V. Assessment of primary colorectal cancer heterogeneity by using whole-tumor texture analysis: contrast-enhanced $\mathrm{CT}$ texture as a biomarker of 5-year survival. Radiology 2013;266(1):177-84.

17. Miles KA, Ganeshan B, Hayball MP. CT texture analysis using the filtration-histogram method: what do the measurements mean? Cancer Imaging 2013;13(3):400-6.

18. Faragher SR, Cleveland RO, Kumar S, Wiseman OJ, Turney BW. In Vitro Assessment of Three Clinical Lithotripters Employing Different Shock Wave Generators. J Endourol 2016;30(5):560-5.

19. Ganeshan B, Miles KA, Young RCD, Chatwin CR. Hepatic enhancement in colorectal cancer: texture analysis correlates with hepatic hemodynamics and patient survival. Acad Radiol 2007;14(12):1520-30.

20. Cui H, Stevens D, Ganeshan B, Turney B. Predicting successful shockwave lithotripsy using CT texture analysis: A potential novel biomarker. European Urology Supplements [Internet] 2016;15(3):e 464. Available from: https://doi.org/10.1016/S1569-9056(16)60466-9

21. Williams JC Jr, Saw KC, Paterson RF, Hatt EK, McAteer JA, Lingeman JE. Variability of renal stone fragility in shock wave lithotripsy. Urology 2003;61(6):1092-6; discussion 1097.

22. Christiansen FE, Andreassen KH, Osther SS, Osther PJS. Internal Structure of Kidney Calculi as a Predictor for Shockwave Lithotripsy Success. J Endourol 2016;30(3):323-6.

23. Miles KA, Ganeshan B, Griffiths MR, Young RCD, Chatwin CR. Colorectal cancer: texture analysis of portal phase hepatic CT images as a potential marker of survival. Radiology 2009;250(2):44452.

24. Chen SH, Ganeshan B, Fraioli F,. Reproducibility of CT Texture Parameters by Leveraging Publicly Available Patient Imaging Datasets. Paper presented at European Congress of Radiology; Vienna, 
Austria; March 2013.

25. Gourtsoyianni S., Ljungqvist G., Khan A., Glynne-Jones R., Ganeshan B., et al. Reproducibility of MRI texture analysis in primary rectal cancer. Paper presented at Radiological Society of North America Annual Meeting; Chicago, America; November 2016. 